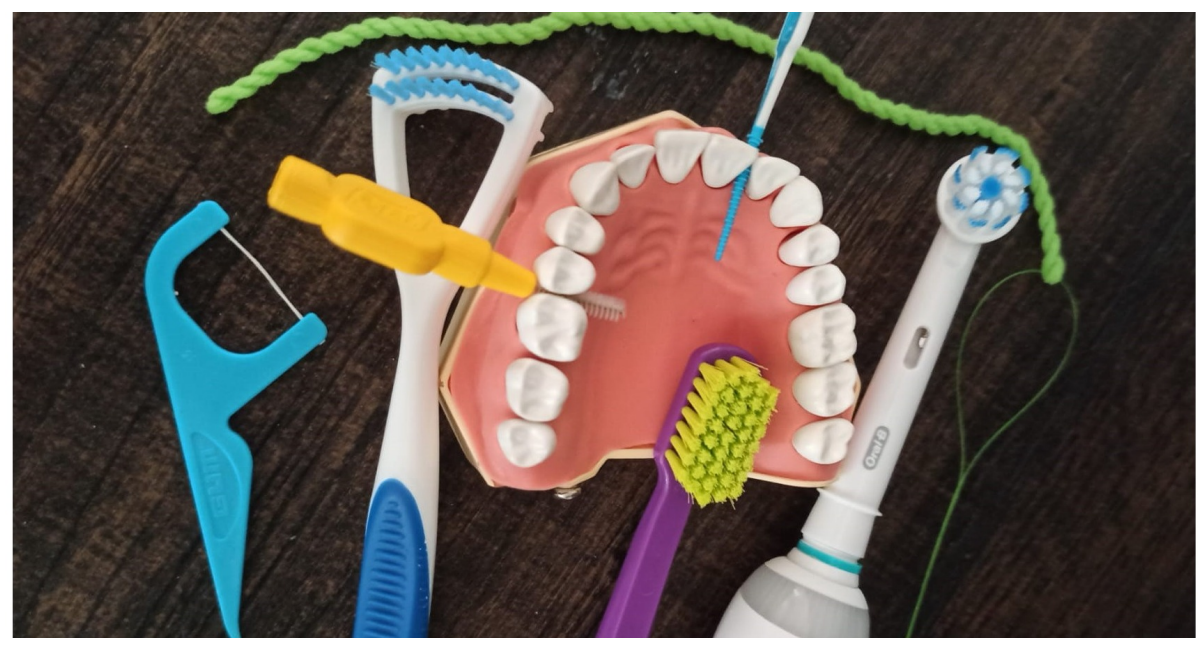

\title{
A primary prevention strategy: oral hygiene and correct lifestyles among patients at risk of developing drug-related osteonecrosis of the Jaw
}

\author{
Giovanna Mosaico, Cinzia Casu
}

Funding: The author(s) received no specific funding for this work.

Potential competing interests: The author(s) declared that no potential competing interests exist.

\section{Abstract}

Skeletal complications caused by osteoporosis or bone metastases are associated with considerable pain, increased mortality and reduced quality of life. Agents that prevent bone resorption, such as bisphosphonates or denosumab, can reduce the risk of fractures and are widely used in patients with osteoporosis or bone cancer metastases. Drug-related osteonecrosis of the jaw (MRONJ) is a rare but potentially serious adverse event associated with cumulative high doses of bisphosphonates or denosumab. MRONJ is a known potential side effect of long-term bisphosphonate therapy. Other risk factors for osteonecrosis of the jaw (ONJ) include the use of glucocorticoids, maxillary or mandibular bone surgery, poor oral hygiene, chronic inflammation, diabetes mellitus, dental prostheses, and other medications, including antiangiogenic agents. Prevention strategies for elimination and stabilization of oral disease include completion of necessary oral surgery prior to initiation of antiresorptive drug therapy, use of antibiotics before and / or after the procedure, and rinsing of the mouth with chlorhexidine antimicrobial, adequate wound healing after tooth extraction and maintenance of good oral hygiene. The primary goal of treatment should be to improve the patient's quality of life by managing pain and infections, preventing the development of new lesions and slowing the progression of the disease. As a multi-professional team, dentists and dental hygienists play a key role in the primary prevention of MRONJ. However, the need for a standardized multidisciplinary approach, with a sustained dialogue between the specialists 
involved, should always be adopted in order to improve the effectiveness of preventive strategies and improve the patient's quality of life.

This treatise describes the preventive treatment and management of patients with this condition.

\section{Background:}

$\mathrm{ONJ}$ is an adverse event of therapy with antiresorptive drugs such as bisphosphonates and denosumab. MRONJ is a relatively rare but potentially serious and debilitating complication. It consists in the progressive bone destruction in the maxillofacial area of patients undergoing treatment with anti antiresorptive drugs, in the absence of a previous radiotherapy treatment. Diagnosis of MRONJ is based on the patient's medical history and in particular on the long-term use of bisphosphonates or denosumab and on the clinical and radiological characteristics of progressive bone destruction (both exposed and unexposed). Possible signs and symptoms of MRONJ are: exposed necrotic bone, sinus tract / fistula, recurrent abscesses, gum swelling, bad breath, loosening of the teeth, mandibular fracture, sinus pain, numbness / dysesthesia of the lower lip.

The diagnosis of ONJ is considered in cases where exposed bone in the maxillofacial region does not heal within 8 weeks in a patient previously treated with an antiresorptive agent. In patients with osteoporosis, ONJ is reported as a very rare adverse event while in cancer patients with bone metastases or malignant hypercalcaemia the incidence is significantly higher (up to $1-10 \%$ of patients). The pathophysiology of $\mathrm{ONJ}$ is not yet fully understood but it is multifactorial. The oral microbiota and dental infections are believed to be central to the development of MRONJ. Prevention is of paramount importance especially in cancer patients.

Since initial MRONJ reporting fifteen years ago, attention has been focused on the association between tooth extraction and adverse events in patients who were already being treated with ONJ-related drugs. More recently, the presence of infection in the dental-periodontal and peri-implant sites has been highlighted as one of the main local risk factors of MRONJ development, often being the main reason for surgical procedures for tooth extraction or implant removal. The link between periodontal disease and the development of MRONJ has been extensively demonstrated and the spread of bacteria through periodontal pockets is one of the main transmission mechanisms of infection through the alveolar bone. Indeed, it is not only the presence of Porphyromonas gingivalis in the periodontal pockets, but also the lgG products that probably promote the development of MRONJ.

The concomitant action of Porphyromonas gingivalis and lgG products would appear to increase bone remodeling and produce pro-inflammatory effects, thus reducing the healing of periodontal tissue and encouraging the development of MRONJ. Therefore, severe periodontitis will be decisive for a poor prognosis for the teeth, for which the only resolution is extraction; The latter has already been discussed as an additional trigger for the development of MRONJ.

Primary prevention, whose main aim is the elimination of oral and dental risk factors, is targeted at restoring and/or maintaining good oral health and reducing the risk of an onset of pathological conditions or any other negative event. This approach has the greatest impact when aimed at protecting constantly the patient's oral health, which is at risk of MRONJ by virtue of controlling local related risk factors.

\section{Materials and Methods:}


We reviewed the literature and guidelines to summarize a practical guide for oral health professionals on managing and maintaining oral health in MRONJ patients.

Scientific articles available on the main medical databases were searched - PubMed, Gogle scolar, Scopus - for which inclusion and exclusion criteria were outlined.

More specifically, the inclusion criteria were:

- Scientific articles published without time limits

- International articles in English and articles in Italian

- Expert review

- Literature reviews

- Narrative reviews

- Historical reviews and key documents (consultation only)

The exclusion criteria were:

- Scientific papers that did not deal with primary prevention and oral hygiene in patients with MRONJ.

\section{Results:}

By entering the keywords "MRONJ diagnosis and prevention" 58 results were obtained, after the preliminary identification, the articles were examined to evaluate the inclusion criteria and 8 were considered compliant.

In an effort to prevent ONJ, optimization of oral health is emphasized and has been shown to be effective in reducing its risk. Recommendations include completion of necessary oral surgery prior to initiation of antiresorptive drug therapy; administration of antibiotics 2-4 days before and 7-10 days after the procedure; antimicrobial oral rinse; appropriate wound closure after tooth extraction; maintaining good oral hygiene. Therefore, the Task Force recommends that when these patients undergo invasive oral surgery, their antiresorptive therapy is suspended until soft tissue healing has occurred. MRONJ prevention must be started prior to treatment. For low-risk patients, the same recommendations apply to preventative dental visits and to maintaining optimal oral health for the general population. The pre-treatment prevention protocol with antiresorptive drugs requires that the patient be motivated to undergo routine oral checks every 4-6 months and to recognize the possible signs and symptoms of ONJ (Table N 1). During the check-ups, a complete oral-dental examination is performed with evaluation of the mucous membranes and tongue, dental formula, periodontal evaluation, evaluation of the quantity and consistency of saliva and mapping of oral lesions. It is important to instruct the patient to maintain optimal oral health, to instruct him to brush his teeth, gums and tongue properly with a soft-bristled toothbrush and a mild fluoride toothpaste two to four times a day, and to clean the spaces between a tooth and the other with dental floss. During therapy with antiresorptive drugs, advise the patient to rinse their mouth and gargle with a saline solution or water and salt every time they brush their teeth.

Table. N.1: Pre-treatment prevention MRONJ 
Pre-treatment prevention with antiresorbable drugs for risk patients ONJ

Oral checks every 4-6 months
- a complete oral-dental examination is performed with evaluation of the mucous membranes and of the tongue

- dental formula

- compilation / updating of the periodontal record

- evaluation of the quantity and consistency of saliva

- the mapping of oral lesions

- instruct the patient to maintain optimal oral health

Educate the patient to recognize the possible signs and symptoms of ONJ

Routine oral hygiene (Table N. 2): The use of electric and sonic toothbrushes are acceptable if the patient is able to use them without causing trauma. Instruct the patient to brush the teeth 2-3 times a day with the roller method with a soft toothbrush with a small head, synthetic threads, rounded and congruent tips, to be disinfected regularly with $5 \%$ hypochlorite and $0,20 \%$ chlorhexidine and allow to dry in the air. Gently brush the tongue avoiding trauma to the gum tissue and gently clean the interdental spaces with dental floss. Fluoride can be applied to the teeth (also in gel with individual masks).

Oral hygiene for an edentulous patient must be performed every time the prosthesis is removed from the oral cavity; the mouth should be rinsed with water and a non-alcoholic mouthwash. The mucous membrane of the gums, tongue and palate should be cleaned with a toothbrush with extra soft bristles or gauze soaked in mouthwash. The movement must be from the back of the mouth forward. Mechanical cleaning of the mucous membranes is useful not only for cleaning but also for stimulating blood circulation and improving resistance to trauma. The mouthwash for oral rinses can be prepared with sodium bicarbonate (1 teaspoon in $250 \mathrm{ml}$ of physiological saline solution) or with $3 \% \mathrm{H} 2 \mathrm{O} 2$ (10 vol diluted 1: 4). To advise lip care products, such as lanolin cream, to prevent dryness and cracking. To advise viscous concentrated hyaluronic acid gels on mucous membranes to relieve pain and promote healing. For xerostomia, recommend saliva replacement products, they perform long-lasting lubricating, humidifying and protective functions of the oral mucosa. To advise rinses with chlorhexidine $0.12 \%$ to $0.2 \%$ for the management of acute gum desease 2 to 4 times a day, depending on the severity. Advise the patient a diet rich in vitamin content and avoid irritating (hot, spicy) and acidic foods. Motivate the patient to control diabetes and to abstain from smoking and alcohol.

During treatment with ONJ, it is recommended to continue brushing and flossing after meals to keep the mouth clean. Removable dental prostheses should not be worn 24 hours a day to give relief to the gum subjected to the pressure of the prosthetic base, a break of at least eight hours would be necessary (for example at night). When the prosthesis is not inserted in the oral cavity it must be cleaned and dipped in a detergent solution. Once used, empty and clean the container to avoid contamination. Cleaning should be done after every meal or every six hours of stay in the oral cavity. Use a toothbrush with nylon filaments and dish soap or mild soap to remove food residue and biofilm. After cleansing, rinse under running water and soak it in a refreshing mouthwash before putting it back in your mouth. Before going to bed, clean the prosthesis and immerse it completely in a 2\% "Amuchina" solution (trade name of Angelini, based in Rome), concentration of hypochlorite in a more diluted solution ( $5 \mathrm{ml}$ of product in $250 \mathrm{cc}$ of water) leave it immersed for at least $15 \mathrm{~m}$ '. Rinse 
well and immerse it in water with mouthwash or special tablets to obtain a refreshing and anti-odor action until ready to wear it again. Before putting it in your mouth, rinse with water. Schedule preventive dental visits even in patients at risk of MRONJ even if edentulous or with removable prostheses, in order to intercept and correct any lesions on the oral mucosa. Strengthen motivation to maintain optimal oral health, to control diabetes, quit smoking and schedule routine checkups every 4-6 months. Educate the patient to recognize the possible signs and symptoms of MRONJ including dental pain, swelling, purulent discharge. Non-surgical periodontal therapy is strongly indicated and must be carefully planned in order to regularly remove plaque and tartar and also periodically review the oral health status of patients being treated.

Therefore, it is essential to plan a four-month follow-up period for cancer patients under treatment, and six months for lowrisk patients of BRONJ without underestimating the patient's contribution to maintaining effective oral hygiene at home and MRONJ self-screening.

\section{Table N.2: Oral hygiene and correct lifestyles to prevention of MRONJ}

\section{Maintenance of the dental-mucosal-periodontal state of health}

- Brush 2-3 times a day with the roller method with a soft brush with a small head, synthetic threads, rounded and

Routine oral hygiene care congruent tips, from disinfect regularly with $5 \%$ hypochlorite and $0.20 \%$ chlorhexidine .

- Gently brushing tongue avoiding trauma to the gum tissue

- Gently clean the interdental spaces with dental floss

- The fluoride can be applied to the teeth (also in gel with individual masks).

- The mouth must be rinsed water and mouthwash in non-alcoholic solution.

- The mucous membrane of the gums, tongue and the palate must be cleaned with a toothbrush with extra soft bristles or one gauze soaked in mouthwash. Movement must be from the back of the mouth to the front.

Oral hygiene for an edentulous patient

Prosthesis hygiene
- The mouthwash for oral rinses can be prepared with sodium bicarbonate (1 teaspoon in $250 \mathrm{ml}$ of physiological saline solution) or with $3 \% \mathrm{H} 2 \mathrm{O} 2$ (10 vol diluted 1: 4).

- To advise concentrated viscous gels of hyaluronic acid on the mucous membranes for relieve wound pain and promote healing.

- For dry mouth, to advise salivary substitute products, they perform a lubricating, humidifying, protective activity longlasting oral mucosa.

- Removable dental prostheses do not need to be worn around the clock to donate relief of the gum subjected to the pressure of the prosthetic base, a break of at least eight hours (for example at night) would be necessary.

- When the prosthesis is not inserted in the oral cavity it must be cleaned immersed in a detergent solution. Once used, empty and clean the container to contamination preventing.

- Cleaning should be done after every meal or every six hours permanence in the oral cavity. Use a toothbrush with nylon filaments and dish soap or mild soap to remove food residues and biofilm. After cleaning, rinse under running water and immerse it in one refreshing mouthwash before placing it back in your mouth.

- Before going to bed, clean the prosthesis and immerse it completely in one $2 \%$ Amuchina solution (5 ml of product in $250 \mathrm{cc}$ of water) eleave it immersed for at least $15 \mathrm{~m}$ :

- Rinse well and soak it in warm water with mouthwash or special tablets to get an action refreshing and anti-odor until it's time to wear it again. Before putting it in your mouth, rinse.

Advise the patient on a diet rich in content vitamins and avoid irritating (hot, spicy) and acidic foods. Motivate the patient to control diabetes and to abstain from smoking and alcohol

Non-surgical periodontal therapy can be performed if there are no major oral lesions that limit the opening of the mouth; there is a number of neutrophils $>2,000 \mathrm{~mm} 3$ and a number of platelets $>40,000 \mathrm{~mm}$. The professional oral hygiene program consists in the compilation / updating of the periodontal record - recording of the probing depth and the clinical level of attachment; recessions; mobility; involvement of bifurcations. Evaluation of the patient's oral hygiene, evaluation of 
the plaque index and the bleeding index. Professional oral hygiene: supragingival plaque and tartar removal, with low frequency mechanical instruments; reinforcement and instruction of home oral hygiene (oral cavity and prosthesis) to prevent the risk of periodontal infections.

If MRONJ is suspected, the patient should be referred to an oral and maxillofacial surgeon or a specialized oncology center experienced in MRONJ. Meanwhile, the dentist may prescribe a mouthwash with $0.12 \%$ chlorhexidine and / or amoxicillin with clavulanic acid 500/125 mg three times a day to treat any related infections.

\section{Conclusions:}

The number of cancer and non-cancer patients treated with ONJ-related drugs and, therefore, the number of potentially adverse events appears to be steadily increasing. It has recently been confirmed that prevention of MRONJ means applying the correct protocols of primary prevention for both pre-treatment and treatment patients, and secondary prevention (i.e., early diagnosis of MRONJ), the Consensus Conference believes that these principles they should be followed by key figures such as doctors, drug prescribers, dentists and oral hygienists. In order to improve the efficacy of therapeutic strategies and increase the patient's quality of life, a standardized multidisciplinary approach should be adopted.

\section{[1][2][3][4][5][6][7]}

[8]

\section{References}

1. 'Olga Di Fede, Vera Panzarella, Rodolfo Mauceri, Vittorio Fusco, et al. (2018). The Dental Management of Patients at Risk of Medication-Related Osteonecrosis of the Jaw: New Paradigm of Primary Prevention. BioMed Research International, vol. 2018 , 1-10. doi:10.1155/2018/2684924.

2. 'Ourania Nicolatou-Galitis, Morten Schiødt, Rui Amaral Mendes, Carla Ripamonti, et al. (2019). Medication-related osteonecrosis of the jaw: definition and best practice for prevention, diagnosis, and treatment. Oral Surgery, Oral Medicine, Oral Pathology and Oral Radiology, vol. 127 (2), 117-135. doi:10.1016/j.00oo.2018.09.008.

3. ^Lawrence Drudge-Coates, Tim Van den Wyngaert, Morten Schiødt, H. A. M. van Muilekom, et al. (2020). Preventing. identifying, and managing medication-related osteonecrosis of the jaw: a practical guide for nurses and other allied healthcare professionals. Support Care Cancer, vol. 28 (9), 4019-4029. doi:10.1007/s00520-020-05440-x.

4. 'A. Fassio, F. Bertoldo, L. Idolazzi, O. Viapiana, et al. (2017). Drug-induced osteonecrosis of the jaw: the state of the art. Reumatismo, vol. 69 (1), 9. doi:10.4081/reumatismo.2017.983. 
5. 'Aliya A. Khan, Archie Morrison, David L. Kendler, Rene Rizzoli, et al. (2017). Case-Based Review of Osteonecrosis of the Jaw (ONJ) and Application of the International Recommendations for Management From the International Task Force on ONJ. Journal of Clinical Densitometry, vol. 20 (1), 8-24. doi:10.1016/j.jocd.2016.09.005.

6. ^M. Migliario, A. Melle, P.L. Foglio Bonda. (2010). Bisfosfonati e osteonecrosi dei mascellari:protocolli clinici per igienisti dentali. Prevenzione \& Assistenza Dentale, vol. 36 (2), 42-48. doi:10.1016/j.pad.2009.10.002.

7. '7-National Cancer Institute. Oral and Dental Management Prior to Cancer Therapy and Management Following Cancer Therapy.

8. ' Giuseppina Campisi, Rodolfo Mauceri, Francesco Bertoldo, Giordana Bettini, et al. (2020). Medication-Related Osteonecrosis of Jaws (MRONJ) Prevention and Diagnosis: Italian Consensus Update 2020. IJERPH, vol. 17 (16), 5998. doi:10.3390/ijerph17165998. 